\title{
Quality of life and informal care burden associated with duchenne muscular dystrophy in Portugal: the COIDUCH study
}

\author{
Valeska Andreozzi ${ }^{1}$, Pedro Labisa ${ }^{1}$, Melina Mota ${ }^{1 *}{ }^{*}$, Susana Monteiro ${ }^{1}$, Rita Alves ${ }^{1}$, João Almeida', \\ Björn Vandewalle1, Jorge Felix , Katharina Buesch², Hugo Canhão ${ }^{3}$ and Igor Beitia Ortiz de Zarate ${ }^{3}$
}

\begin{abstract}
Background: To describe the reduced health-related quality of life (HRQoL) of duchenne muscular dystrophy (DMD) patients and their caregiver burden and to present its relationship with disease progression.

Methods: This cross-sectional study assessed patient HRQoL with the 3-level version of the EuroQol-5D (EQ-5D-3L) and caregiver burden with the Work Productivity and Activity Impairment: General Health questionnaire. DMD patients and their caregivers were identified through Portuguese Neuromuscular Association (APN).

Results: A total of $46 \mathrm{DMD}$ main caregivers, of eight ambulant and 38 non-ambulant patients, completed the questionnaires. Over half (58.7\%) of all non-ambulant patients were on ventilation support, either full-time (15.2\%) or non full-time (43.5\%). Non-ambulant patients had a lower mean utility scores than ambulant patients ( -0.05 versus $0.51, p$ value $<0.001$ ). Caregivers of non-ambulant patients reported a significant mean daily activity impairment as compared to caregivers of ambulant patients ( $68 \%$ versus $23 \%, p$ value $<0.001)$. Among non-ambulant patients, both utility scores and caregiver impairment appeared to deteriorate according to a higher need for ventilation support, however, these results were not statistically significant.
\end{abstract}

Conclusions: These results emphasise the significant negative impact that DMD progression has on the patient HRQoL, as well as caregivers' ability to conduct their daily activities. Therapeutic options that stop or slow the disease progression could have a beneficial impact for both patients and caregivers.

Keywords: Duchenne muscular dystrophy, Disease progression, Quality of life, Informal care burden

\section{Background}

Duchenne Muscular Dystrophy (DMD) is a rare X-linked disorder which leads to the progressive degeneration of muscle tissues due to the lack or deficiency of the protein dystrophin [1]. First symptoms appear around the age of four, when diagnosis is typically established [2].

In the natural course of the disease, lower limb function is the first to be affected, and as patients age, muscular

\footnotetext{
*Correspondence: melina.mota@exigoconsultores.com

${ }^{1}$ Exigo Consultores, Lisbon, Portugal

Full list of author information is available at the end of the article
}

damage tends to accumulate, leading to an increasing level of symptom severity and lower function scores [3, 4]. Loss of ambulation occurs between the ages of 8 and 14 years [3] and precedes the development of other pulmonary and cardiac complications, which constitute the main causes of death [5].

There is no cure for DMD [1]. A combination of nonpharmacological treatments (physical therapy, surgery, ventilation support) and pharmacological treatments (glucocorticoids, angiotensin-converting-enzyme [ACE] inhibitors, beta-blockers and, in nonsense mutation DMD patients, ataluren) have helped delay disease original author(s) and the source, provide a link to the Creative Commons licence, and indicate if changes were made. The images or other third party material in this article are included in the article's Creative Commons licence, unless indicated otherwise in a credit line to the material. If material is not included in the article's Creative Commons licence and your intended use is not permitted by statutory regulation or exceeds the permitted use, you will need to obtain permission directly from the copyright holder. To view a copy of this licence, visit http://creativecommons.org/licenses/by/4.0/. The Creative Commons Public Domain Dedication waiver (http://creativeco mmons.org/publicdomain/zero/1.0/) applies to the data made available in this article, unless otherwise stated in a credit line to the data. 
progression and alleviate symptom severity $[1,6]$. Altogether this multiapproach comprehensive care has contributed to a substantial increase in median lifeexpectancy at birth, which now reaches up to 40 years of age [7].

Improved medical care practices also had a positive impact on health-related quality of life (HRQoL). More advanced disease stages, characterized by the loss of ambulation or presence of ventilation support, have typically been associated with a lower HRQoL [8]. In addition to the direct benefits of delaying disease progression, such as loss of ambulation at a later age $[1,6]$, a better management of symptom severity allows patients to sustain a higher HRQoL for much longer than they otherwise would [9].

Caregiver burden is also a great concern to most families. Patients require a high number of informal care hours, which has a detrimental effect on the mental and physical health of caregivers, as well as on their day-today activities and professional life [10]. Yet, to our knowledge, there is a lack of data assessing daily caregiver activity impairment according to the disease stage of the patients under their care.

The COIDUCH study aimed to assess the cost of illness (COI), HRQoL of patients and the burden to caregivers associated with DMD, in Portugal. This paper reports on the HRQoL of patients and caregiver burden, and presents their relationship with disease progression.

\section{Methods}

\section{Study design and data collection}

The COIDUCH study was a cross-sectional study that included patients with DMD and their caregivers. To be included, patients were required to have a DMD diagnosis and be registered at the Portuguese Neuromuscular Association (APN). Any patients receiving experimental drugs or placebo in randomized clinical trials were excluded.

Face-to-face interviews were conducted by trained interviewers between June and August, 2019. After written informed consent, caregivers answered a customized questionnaire. The questionnaire collected patient and main caregiver data on demographic and clinical characteristics, i.e., age, sex, diagnosis date, ambulatory and assisted ventilation status, caregiver relationship to the patient, and informal care provided.

Patient HRQoL was assessed with the 3-level version of the EuroQol-5D (EQ-5D-3L) proxy version 2 instrument [11], in which caregivers were asked to rate how they thought the patients under their care would rate their own HRQoL, were they able to do so. Caregiver burden was measured with the Work Productivity and Activity Impairment: General Health (WPAI:GH) questionnaire [12], which assesses the impact health problems have on work productivity and regular daily activity.

All patients and caregivers were informed about the study objectives and data confidentiality.

\section{HRQoL and caregiver burden measures}

The EQ-5D-3L instrument comprises a descriptive system based on five dimensions of health status: mobility, self-care, usual activities, pain/discomfort and anxiety/ depression [13]. Each dimension in the descriptive system has three levels of response (no problems, moderate problems, or extreme problems). A validated translation of this instrument to Portuguese was used [13]. The EQ5D-3L descriptive index responses were mapped into a single dimension HRQoL utility value ranging from full health (utility $=1$ ), over death (utility $=0$ ) to health states perceived as worse than death (utility $<0$ ), using validated Portuguese population norms [14].

The WPAI:GH questionnaire [12] is a validated instrument and consists of six items covering employment status, hours missed from work due to health problems, hours missed from work due to other reasons, hours actually worked, how health problems affect productivity at work and how health problems affect regular daily activities. The questionnaire was adapted as to reflect the burden to the caregiver of the DMD patient under their care. The six questions were used to derive four domains: work time missed (absenteeism), impairment while working (presenteeism), overall work impairment (combining absenteeism and presenteeism) and activity impairment (daily activities). WPAI:GH outcomes on all four domains are expressed as impairment percentages $(0-100 \%)$, with higher numbers indicating greater impairment and less productivity [15].

\section{Statistical analysis}

Summary statistics were calculated for the overall population demographic, clinical, EQ-5D-3L and WPAI:GH data, as means and standard deviations (SD) for continuous data and absolute numbers and relative frequencies for categorical data.

Disease progression was evaluated according to the ambulatory status and/or ventilation support of patients. The tests of the null hypothesis of no difference on EQ-5D-3L utility and WPAI daily activity impairment according to ambulatory status and ventilation support were performed by the Wilcoxon and Kruskal-Wallis tests, respectively.

All analyses were performed using the R statistical software, considering a significance level of 5\% [16]. 


\section{Results}

A total of 53 patient/caregiver pairs identified by APN were invited to take part in the study. Of those, three did not meet the inclusion criteria and four declined to participate. Therefore, 46 patient/caregiver pairs met the inclusion criteria and were available to participate in the study.

All DMD patients were male, with a mean age of 18.9 $(\mathrm{SD}=8.2)$ years. Of these, $38(82.6 \%)$ were non-ambulant, having lost ambulation at a mean age of $9.7(\mathrm{SD}=2.3)$ years. Over half $(58.7 \%)$ of all non-ambulant patients were on ventilation support, either full-time (15.2\%) or non full-time (43.5\%).

The main caregivers reported a mean of $12.6(\mathrm{SD}=5.6)$ hours of daily informal care and were mostly parents to the DMD patient (Table 1).

Table 1 Characteristics of the study participants

\begin{tabular}{ll}
\hline & $\begin{array}{l}\text { Total cohort } \\
\mathbf{( N = 4 6 )}\end{array}$ \\
\hline Patients & \\
Mean age, years & $18.9 \pm 8.2$ \\
Mean age at diagnosis, years & $4.4 \pm 2.4$ \\
Non-ambulant patients, n (\%) & $38(82.6)$ \\
Mean age at loss of ambulation, years & $9.7 \pm 2.3$ \\
Patients on assisted ventilation, n (\%) & $27(58.7)$ \\
Full-time & $7(15.2)$ \\
Non full-time & $20(43.5)$ \\
Mean age at start of assisted ventilation, years & $16.9 \pm 4.3$ \\
Caregiver & \\
Female, $n$ (\%) & $39(84.8)$ \\
Mean age, years & $48.8 \pm 9.8$ \\
Relationship to the patient, $\mathrm{n}(\%)$ & \\
Parent & $44(95.7)$ \\
Other family member & $2(4.3)$ \\
Number of daily informal care hours & $12.6 \pm 5.6$ \\
\hline
\end{tabular}

Data presented as mean $\pm \mathrm{sd}$ or $\mathrm{n}(\%)$. Because of rounding, percentages might not add up to exactly $100 \%$

a Only the main caregiver data was included

\section{Quality of life}

Table 2 reports summary statistics on the main caregiver assessment of how patients would rate their own HRQoL. With exception to anxiety/depression, the majority of patients were rated as having either moderate or extreme problems in all EQ-5D-3L dimensions. The highest prevalence of extreme problems was in the self-care (78.3\%) dimension, followed by mobility (37.0\%) and usual activities (34.8\%).

Overall, DMD patients had a low HRQoL, with a mean utility value of $0.05(\mathrm{SD}=0.33)$. The presence of negative utility values was higher among non-ambulant patients $(61 \%)$, with a mean utility value of $-0.05(\mathrm{SD}=0.24)$, while entirely absent in ambulant patients, which had a mean utility value of 0.51 ( $\mathrm{SD}=0.28)$. As shown in Fig. 1 , the difference in utility values according to ambulatory status was statistically significant ( $p$ value $<0.001)$.

Figure 2 presents boxplots of the utility values and the mean utilities among non-ambulant patients according to ventilation support. Patients with full-time ventilation support presented the lowest mean utility value $(-0.20$; $\mathrm{SD}=0.22)$, followed by patients with non full-time ventilation support $(-0.05$; $\mathrm{SD}=0.21)$ and no ventilation support $(0.03 ; \mathrm{SD}=0.29)$. Although data suggest a decline of HRQoL utility values while the need for ventilation support increases, statistical significance was not reached ( $p$ value $=0.228)$.

The presence of negative utilities was higher in fulltime $(86 \%)$ and non full-time ventilated (60\%) patients, slightly less so in patients with no ventilation support $(45 \%)$. There were, however, no statistically significant difference between groups ( $p$ value $=0.233$ ).

\section{Work productivity and activity impairment}

A total of 15 (32.6\%) caregivers were employed at the time of interview. Of these, 12 had worked in the 7 days prior to the interview and were able to respond to the absenteeism and presenteeism related domains of the WPAI:GH questionnaire. Despite a low level of reported impact on absenteeism (11.1\% in terms of work time

Table 2 Caregiver assessment of the patient 3-level response distribution of the five domains in the EQ-5D-3L questionnaire

\begin{tabular}{|c|c|c|c|c|c|}
\hline & \multicolumn{5}{|c|}{$\begin{array}{l}\text { EQ-5D-3L dimensions } \\
(\mathrm{N}=46)\end{array}$} \\
\hline & Mobility & Self-Care & Usual activities & Pain and discomfort & $\begin{array}{l}\text { Anxiety } \\
\text { and } \\
\text { depression }\end{array}$ \\
\hline No problems & $1(2.2)$ & $4(8.7)$ & $5(10.9)$ & $15(32.6)$ & $26(56.5)$ \\
\hline Moderate problems & $28(60.9)$ & $6(13.0)$ & $25(54.3)$ & $30(65.2)$ & $19(41.3)$ \\
\hline Extreme problems & $17(37.0)$ & $36(78.3)$ & $16(34.8)$ & $1(2.2)$ & $1(2.2)$ \\
\hline
\end{tabular}

Data presented as $\mathrm{n}(\%)$; Because of rounding, percentages might not add up to exactly $100 \%$ 


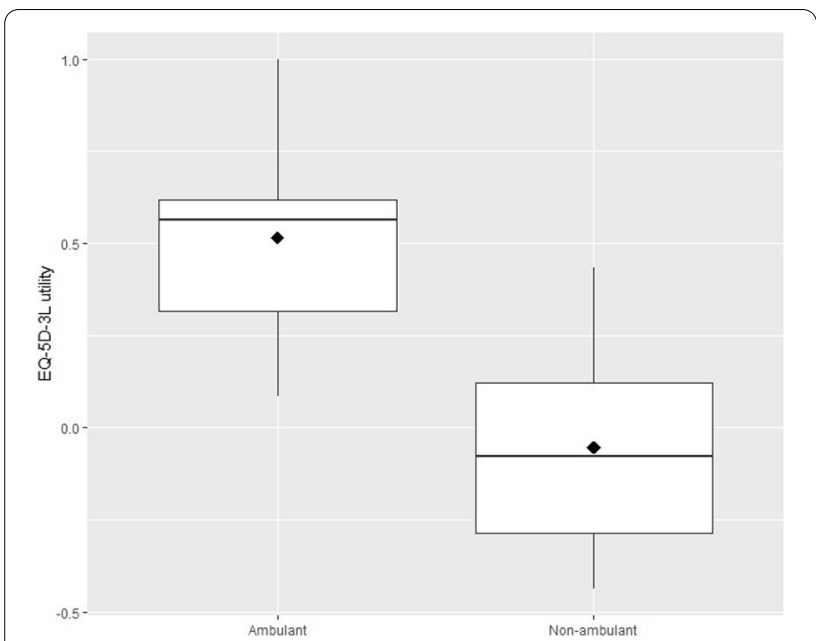

Fig. 1 Box-plots of utility values in ambulant and non-ambulant patients. Diamond $=$ mean value

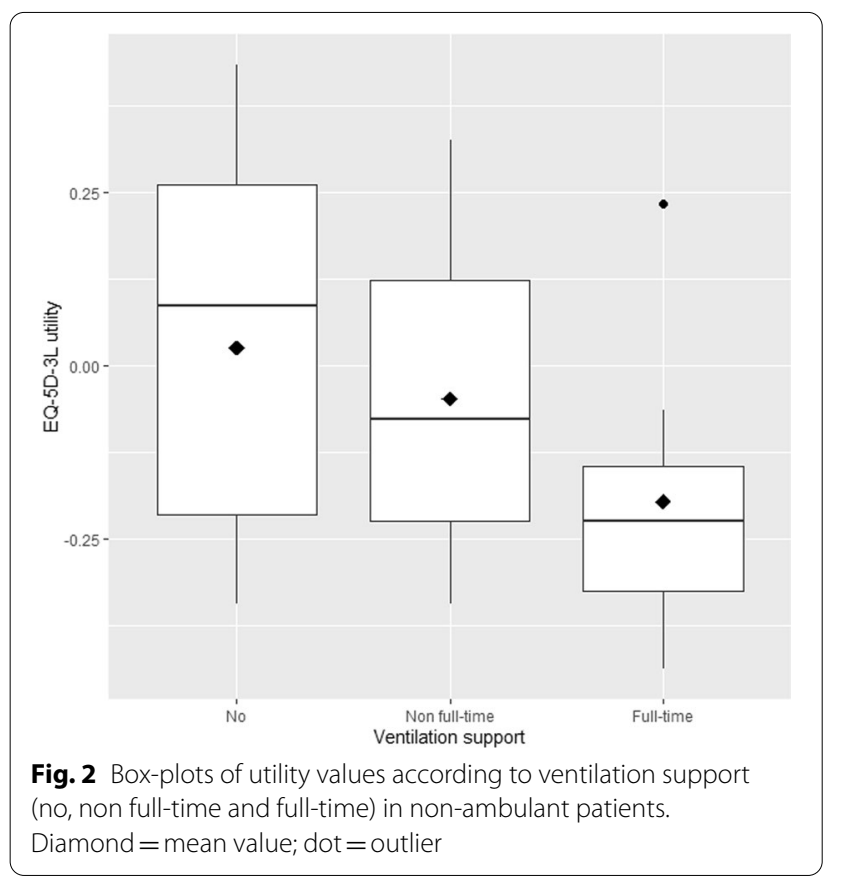

missed), there was a relatively high level of impact of caring duties on presenteeism (35.8\% impairment while working), leading to an overall level of work impairment of $30.5 \%$ (Table 3 ).

Considering all 46 caregivers interviewed, the mean reported daily activity impairment was 60\% (Table 3). Caregivers of non-ambulant patients reported a mean daily activity impairment of $68 \%$, a value significantly higher as compared to caregivers of ambulant patients, which reported a mean value of $23 \%$ ( $p$ value $<0.001)$ (Fig. 3).

Caregivers of non-ambulant patients with either fulltime or non full-time ventilation support presented a higher daily activity impairment $(77 \%$ and $71 \%$, respectively), compared to patients without ventilation support (57\%), but there were no statistically significant differences between groups ( $p$ value $=0.173)$ (Fig. 4).

\section{Discussion}

To the best of our knowledge this was the first study to assess both DMD patient HRQoL and caregiver burden according to disease stage, measured by the EQ5D-3L and WPAI:GH instruments, respectively. Given its known and predictable progression pathway, disease severity in DMD patients is usually categorized according to functional stages, related to ambulatory status, as well as other relevant clinical factors, such as the need for ventilation support.

Our results confirm that loss of ambulation is an important clinical event, that occurs early in life, around the age of 10 , and is associated with a substantial impact in HRQoL. The mean utility in non-ambulant patients was -0.05 , significantly lower than that of ambulant patients (0.51). In non-ambulant patients there was also a trend towards lower utility values, as the need for ventilation support, expressed by frequency of use, increased. However, this trend did not prove to be statistically significant.

These results are consistent with those in other studies which have shown lower health utility values associated with non-ambulation, as well as more advanced

Table 3 Caregiver work productivity impairment (WPAI:GH) scores (\%)

\begin{tabular}{lr}
\hline & Mean \pm SD \\
\hline Caregivers who worked in the past 7 days $(n=12)$ & $11.1 \pm 13.1$ \\
$\quad$ Percent work time missed due to patient's DMD (absenteeism) & $35.8 \pm 22.8$ \\
$\quad$ Percent impairment while working due to patient's DMD (presenteeism) & $30.5 \pm 19.0$ \\
$\quad$ Percent overall work impairment due to patient's DMD (combining absenteeism and presenteeism) & $60 \pm 32.2$ \\
All caregivers ( $n=46)$ & \\
$\quad$ Percent activity impairment due to patient's DMD (daily activities)
\end{tabular}




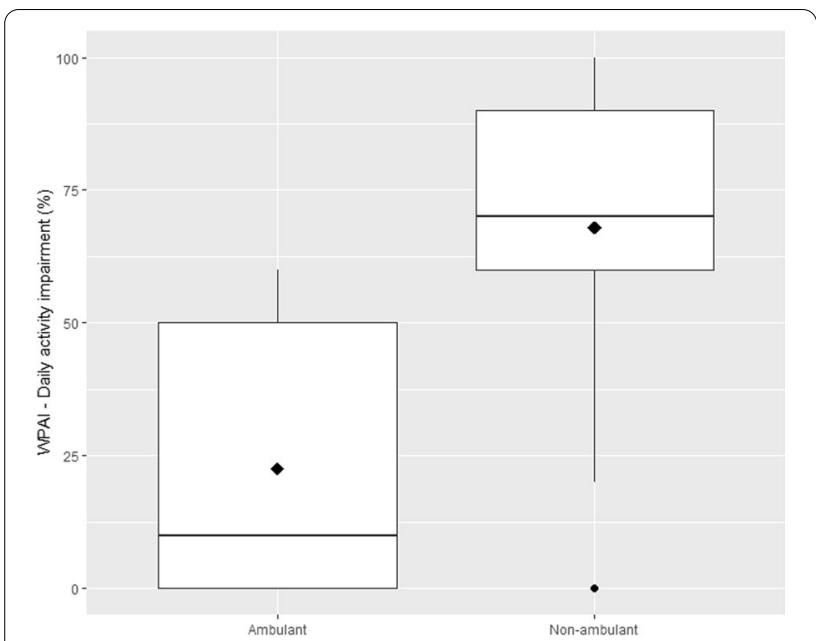

Fig. 3 Box-plots of daily activity impairment in caregivers of ambulant and non-ambulant patients. Diamond = mean value; dot $=$ outlier

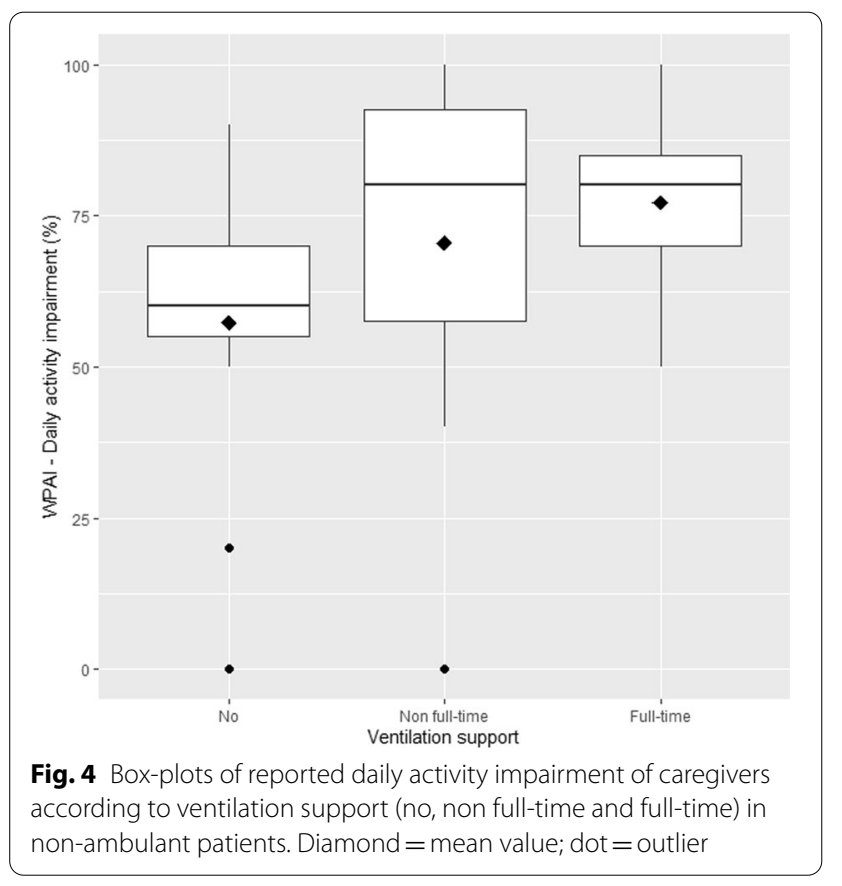

disease stages, such as full-time ventilation support [17-20]. One distinct feature, however, relates to the presence of negative mean utility values in non-ambulant patients, suggesting that these patients may be seen by society as being in a health state worse than death. This diverges from most studies which have suggested low, but positive, utility values in DMD patients, including those in late-stage disease [8]. One exception being Cavazza et al. [18], in which adult DMD patients from Spain, Sweden and United Kingdom reported negative utilities, while also using the EQ-5D-3L questionnaire.

One possible explanation resides in the use of different country specific value sets, making this heterogeneity in utilities a reflection of different societal perceptions for the same health states. However, it remains unclear to which extent other factors, such as patient heterogeneity or perspective used to assess the patients' HRQoL (patient vs caregiver reporting), may have contributed to this discrepancy in results.

Our results also show that disease progression has a substantial impact on caregiver burden, assessed by the percentage of caregiver daily activity impairment. Caregivers of non-ambulant patients reported a mean daily activity impairment of $68 \%$, close to three times that of caregivers of ambulant patients (23\%), with a statistically significant difference between both groups ( $p$ value $<0.001$ ). There was also a trend towards higher impairment levels in caregivers of non-ambulant patients with higher need for ventilation support, but this trend did not reach statistical significance. As disease severity increases, caregivers allocate an increasing percentage of their time to informal care, which explains the high number of daily informal care hours reported (12.6). This not only limits their ability to retain employment, but also reduces the number of leisure hours left in the day, and is consistent with the results in other published studies, albeit with the use of alternative questionaries $[3,10]$.

The cross-sectional data presented helps understand the consequences that disease progression carries for important patient and caregiver outcomes. Current treatment options, such as corticosteroids, have shown to delay the natural course of disease progression, namely loss of ambulation and respiratory function, and their use is recommended in ambulant patients and, to a lesser extent, in non-ambulant patients as well [1]. However, the results from our study show that despite the potential benefits of the current treatment options, disease progression and its direct consequences, both in terms of utility deterioration and caregiver daily activity impairment, are still a major source of concern. Recent innovative treatment options, such as ataluren, have shown to further delay disease progression in certain groups of patients (nonsense mutation DMD), including important clinical outcomes such as loss of ambulation or pulmonary function [21]. This delay in critical outcomes will likely allow patients to maintain higher utility levels for longer periods of time, as well as decrease caregiver burden. Hence, ensuring conditions for adequate access to innovative treatments for DMD patients appears to be of critical importance.

Moreover, addressing caregiver burden through other measures, such as expanding the financial and social 
support schemes available to affected families, should also be considered. The recent introduction of a formal status for informal caregivers in Portugal, which aims to improve financial support, caregiving training, psychological support and help reconcile patient care duties with professional life, represents an important step forward [22].

In addition to the use of two validated questionnaires which had not been used before in conjunction in this context, another asset of this study resides in the way data was collected, with face-to-face interviews, which helped minimize the risk of questions being misinterpreted, at least when compared to other more indirect methods.

One limitation of this study relates to the potential for referral bias from having the recruitment process of DMD patients relying on a prior membership in the APN. Membership in this association is voluntary and may be more attractive for patients with a more severe clinical presentation of DMD, since it is attached with a greater availability of clinically relevant services, such as rehabilitation.

Another limitation relates to the sample size of patients included in this study. Even though our sample likely represents a large percentage of the expected prevalence of DMD in Portugal, the total number of patients included was still smaller compared to that in other studies $[10,17$, $18,23]$. This may have contributed to the lack of statistical significance in some of the comparisons performed, such as in non-ambulant patients according to the need for ventilation support.

To ensure that the type of HRQoL data was consistent between paediatric and adult patients, EQ-5D-3L was assessed exclusively through the use of caregiver-proxy reporting. Studies have shown that for the same health states, utility data obtained from proxy reporting tends to be lower than that of patient reporting [8]. This is a limitation that may help further explain the high presence of negative utility values among non-ambulant patients in our study.

In addition to the use of a WPAI:GH questionnaire adaptation, which was not validated for the use in DMD, our exclusive focus on main caregiver burden likely resulted in an underestimation of the total caregiver burden, since it neglected the contribution of other family members to the informal care of the patient.

\section{Conclusion}

In conclusion, our results emphasise the impact of DMD on the HRQoL of patients and the burden to their caregivers, which gets increasingly detrimental as disease progresses. This highlights the need for therapeutic options that can stop or slow down disease progression, at any point in the disease history.

\section{Abbreviations}

ACE: Angiotensin-converting-enzyme; APN: Portuguese Neuromuscular Association; COI: Cost of illness; DMD: Duchenne muscular dystrophy; EQ-5D-3L: 3-Level version of the EuroQol-5D; GDPR: General Data Protection Regulation; HRQOL: Health-related quality of life; SD: Standard deviation; WPAI:GH: Work

Productivity and Activity Impairment: General Health.

\section{Acknowledgements}

The authors thank the Portuguese Neuromuscular Patients Association for its assistance in validating the health resource questionnaire as well as in identifying DMD patients.

\section{Authors' contributions}

VA designed the study, analysed the data and drafted the manuscript. PL and MM designed the study, collected the data and contributed to the draft of the manuscript. RA analysed the data. SM and JA collected the data. JF and $\mathrm{BV}$ designed the study and contributed in the interpretation of the data. $\mathrm{KB}$, $\mathrm{HC}$ and $\mathrm{IBOZ}$ supported the planning phase of the interviews and approved the final version to be published. All authors read and approved the final manuscript.

\section{Funding}

This study was funded by PTC Therapeutics. Authors ascribed to Exigo Consultores are employees of an independent consulting firm and maintained independent scientific control over the study, including data analysis and interpretation of final results.

\section{Availability of data and materials}

All aggregate data generated or analysed are included in this published article. No individual data is available to protect the recognition of individual patients.

\section{Declarations}

\section{Ethics approval and consent to participate}

Ethical principles and good clinical research practices guided the execution of this study. Prior written informed consent from the participants was obtained. The anonymity of the participants was guaranteed. All personal data was managed according to the General Data Protection Regulation (GDPR).

\section{Competing interests}

VA, PL, MM, SM, RA, JA, BV and JF are employees of Exigo Consultores. Exigo Consultores is providing consulting services to PTC Therapeutics under a fee for services contract. KB, HC and IBOZ are employees of PTC Therapeutics.

\section{Author details}

${ }^{1}$ Exigo Consultores, Lisbon, Portugal. ${ }^{2}$ PTC Therapeutics International, Steinhausen, Switzerland. ${ }^{3}$ PTC Therapeutics Portugal, Sintra, Portugal.

Received: 22 June 2021 Accepted: 15 February 2022

Published online: 03 March 2022

\section{References}

1. Birnkrant DJ, Bushby K, Bann CM, Apkon SD, Blackwell A, Brumbaugh D, et al. Diagnosis and management of Duchenne muscular dystrophy, part 1: diagnosis, and neuromuscular, rehabilitation, endocrine, and gastrointestinal and nutritional management. Lancet Neurol. 2018;17(3):251-67.

2. Pane M, Scalise R, Berardinelli A, D'Angelo G, Ricotti V, Alfieri P, et al. Early neurodevelopmental assessment in Duchenne muscular dystrophy. Neuromuscul Disord. 2013;23(6):451-5.

3. Ryder S, Leadley RM, Armstrong N, Westwood M, de Kock S, Butt T, et al. The burden, epidemiology, costs and treatment for Duchenne muscular dystrophy: an evidence review. Orphanet J Rare Dis. 2017;12(1):79.

4. Jung I-Y, Chae JH, Park SK, Kim JH, Kim JY, Kim SJ, et al. The correlation analysis of functional factors and age with Duchenne muscular dystrophy. Ann Rehabil Med. 2012;36(1):22-32. 
5. Passamano L, Taglia A, Palladino A, Viggiano E, D'Ambrosio P, Scutifero $\mathrm{M}$, et al. Improvement of survival in Duchenne Muscular Dystrophy: retrospective analysis of 835 patients. Acta Myol. 2012;31 (2):121-5.

6. Birnkrant DJ, Bushby K, Bann CM, Alman BA, Apkon SD, Blackwell A, et al. Diagnosis and management of Duchenne muscular dystrophy, part 2: respiratory, cardiac, bone health, and orthopaedic management. Lancet Neurol. 2018;17(4):347-61.

7. Landfeldt E, Thompson R, Sejersen T, McMillan HJ, Kirschner J, Lochmüller H. Life expectancy at birth in Duchenne muscular dystrophy: a systematic review and meta-analysis. Eur J Epidemiol. 2020;35(7):643-53.

8. Szabo SM, Audhya IF, Malone DC, Feeny D, Gooch KL. Characterizing health state utilities associated with Duchenne muscular dystrophy: a systematic review. Qual Life Res. 2020;29(3):593-605.

9. Pangalila RF, Van Den Bos GA, Bartels B, Bergen M, Stam HJ, Roebroeck ME. Prevalence of fatigue, pain, and affective disorders in adults with Duchenne muscular dystrophy and their associations with quality of life. Arch Phys Med Rehabil. 2015;96(7):1242-7.

10. Landfeldt E, Edström J, Buccella F, Kirschner J, Lochmüller H. Duchenne muscular dystrophy and caregiver burden: a systematic review. Dev Med Child Neurol. 2018;60(10):987-96.

11. Brooks R, Group E. EuroQol: the current state of play. Health Policy. 1996;37(1):53-72.

12. Reilly MC, Zbrozek AS, Dukes EM. The validity and reproducibility of a work productivity and activity impairment instrument. Pharmacoeconomics. 1993;4(5):353-65.

13. EuroQol Research Foundation. EQ-5D-3L user guide 2018. https://euroq ol.org/publications/user-guides.

14. Ferreira LN, Ferreira PL, Pereira LN, Oppe M. EQ-5D Portuguese population norms. Qual Life Res. 2014;23(2):425-30.

15. Reilly Associates. WPAl scoring [19/11/2020]. http://www.reillyassociates. net/WPAl_Scoring.html.

16. R Core Team. R: A Language and Environment for Statistical Computing. R Foundation for Statistical Computing.

17. Landfeldt E, Lindgren P, Bell CF, Guglieri M, Straub V, Lochmüller $H_{\text {, }}$ et al. Health-related quality of life in patients with Duchenne muscular dystrophy: a multinational, cross-sectional study. Dev Med Child Neurol. 2016;58(5):508-15.

18. Cavazza M, Kodra Y, Armeni P, De Santis M, López-Bastida J, Linertová R, et al. Social/economic costs and health-related quality of life in patients with Duchenne muscular dystrophy in Europe. Eur J Health Econ. 2016;17(Suppl 1):19-29.

19. Landfeldt E, Lindgren P, Bell CF, Schmitt C, Guglieri M, Straub V, et al. The burden of Duchenne muscular dystrophy: an international, cross-sectional study. Neurology. 2014;83(6):529-36.

20. Pangalila RF, van den Bos GAM, Stam HJ, van Exel NJA, Brouwer WBF, Roebroeck ME. Subjective caregiver burden of parents of adults with Duchenne muscular dystrophy. Disabil Rehabil. 2012;34(12):988-96.

21. Mercuri E, Muntoni F, Osorio AN, Tulinius M, Buccella F, Morgenroth $L P$, et al. Safety and effectiveness of ataluren: comparison of results from the STRIDE Registry and CINRG DMD Natural History Study. J Comp Eff Res. 2020;9(5):341-60.

22. Lei n.o 100/2019 da Assembleia da República, Diário da República n.o 171/2019, Série I; 2019. https://data.dre.pt/eli/lei/100/2019/09/06/p/dre.

23. Schreiber-Katz O, Klug C, Thiele S, Schorling E, Zowe J, Reilich P, et al. Comparative cost of illness analysis and assessment of health care burden of Duchenne and Becker muscular dystrophies in Germany. Orphanet J Rare Dis. 2014;9:210.

\section{Publisher's Note}

Springer Nature remains neutral with regard to jurisdictional claims in published maps and institutional affiliations. 